\title{
Deauville score: the Phoenix rising from ashes
}

\author{
Egesta Lopci ${ }^{1}$ (1) $\cdot$ Michel Meignan ${ }^{2}$
}

Received: 31 October 2018 / Accepted: 7 November 2018 / Published online: 14 November 2018

(C) Springer-Verlag GmbH Germany, part of Springer Nature 2018

The biggest success for medical imaging arrives when the findings therein provided have clinical usefulness and directly impact patients' management and outcome. Similar aims should always lead the work of imagers and particularly ours in nuclear medicine. Hence, the significant results obtained from important clinical trials proving the relevance of positron emission tomography (PET)-guided treatment in patients, such as HD18 or AHL2011 in advanced-stage Hodgkin's lymphoma $[1,2]$ appear exceptional and "world breaking", although they should be the natural modus operandi in molecular imaging research. Based on linear concepts and "simple" reading with Deauville score [3], the results of these trials prove what is known as the current paradigm of response in Hodgkin's lymphoma (Fig. 1): after two cycles of chemotherapy, metabolic response on PET is already able to predict the outcome of the patient and can be used to adapt therapeutic intensity accordingly [4]. This concept took time before being endorsed by the medical community. It now finds arguments in the extensive literature published in the last decade and pioneered by nuclear medicine physicians and medical oncologists working together. The current paradigm is based on several premises: 1) imaging response is assessed based on metabolism and not only on morphology; 2) international criteria for response help discriminate the different pathophysiological meanings of residual uptake; 3 ) the best moment to assess response is defined in order to change efficiently the therapeutic strategy $[5,6]$. Yet, the evidence for this paradigm of response now appears clearly demonstrated for first-line treatment and in Hodgkin's lymphoma. Does it fit as a reference model for other lines, or even in case of immunemodulatory regimens?

Egesta Lopci

egesta.lopci@gmail.com; egesta.lopci@cancercenter.humanitas.it

1 Nuclear Medicine Department, Humanitas Clinical and Research Hospital, Via Manzoni 56, 20089 Rozzano, MI, Italy

2 Radiology, Lysa Imaging and Haematology, Hôpitaux Universitaires Henri Mondor, Créteil, France
At a first, superficial sight, the model might not fit. The worldwide "bugbear" of immune-related side effects and associated risk of false positive uptake of $18 \mathrm{~F}$ fluorodeoxyglucose (FDG) have markedly limited the use of FDG PET in immunotherapy trials, particularly with the advent of checkpoint inhibitors, in Hodgkin's disease. It seems as if the history of what is done for chemotherapy has been forgotten, and so it seems also for the functional value of PET results. We must not forget, anyhow, that FDG uptake in a Hodgkin's tumor at staging is by its very nature "false positive", since tumor lesions are composed by only $1 \%$ ReedSternberg (RS) cells, which "dive" in a vast ocean of immune infiltrate. Hence, the high metabolism documented is nothing but the result of the interrelationship between these solitary RS cells with the tumor microenvironment, and the so-called "pseudo-progression" under checkpoint inhibitors should not surprise. It translates in FDG uptake the effect of the drug on the immunocompetent system and should be used to help understand the phenomenon. Notwithstanding, some criteria have been built in an attempt to classify the different types of response (LYRIC) [7]. Others came back to old RECIST-type analysis (RECIL) reducing the role of PET [8]. This limitation of the PET use with checkpoint inhibitors is clear when performing a PubMed research for: "PET", or "immunotherapy", or "Hodgkin", or "lymphoma". Only three original articles appear pertinent to the argument for checkpoint inhibitors, all retrospective [9-11], with one moreover analyzing chemotherapy or chemo-anti-PD-1 combination after failed anti-PD-1 therapy [11]. Nevertheless, the common trait d'union for the manuscripts is the predictive potential of metabolic response assessment with Deauville score already after a few cycles of immunotherapy (Fig. 2). The papers quote the 5-point scale after 2-3 months of anti-PD-1 (either nivolumab or pembrolizumab) as predictive of subsequent response 4 6 months later $[9,10]$. Moreover, the classification of patients into responders (Complete Response + Partial Response) or non-responders (Stable Disease + Progressive Disease) to immunotherapy could correlate significantly with progressionfree survival of the cohort [10]. Yet, when the common models of response to therapy seem to fail in new immune-modulatory 
DIAGNOSIS

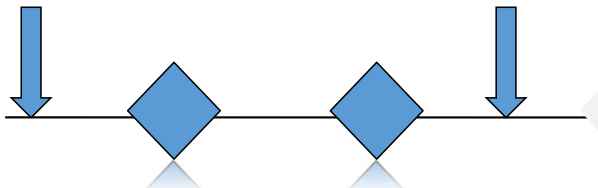

\section{STAGING}

Fig. 1 Current paradigm of response to therapy in Hodgkin's lymphoma. Based on the type of response at interim evaluation, patients showing a complete metabolic response (Deauville Score: DS 1, 2, 3) are more likely to have a complete response to treatment and a better outcome than patients without (DS 4, 5). The two cases herein display different patterns of response: patient \#1 (upper panels) shows a partial metabolic

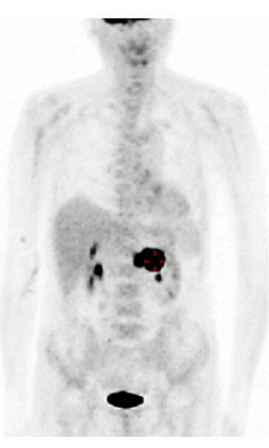

STAGING

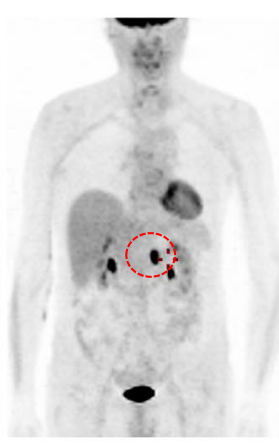

INTERIM

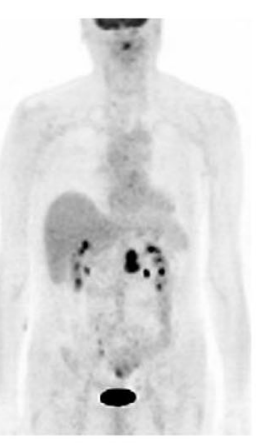

END-TREATMENT

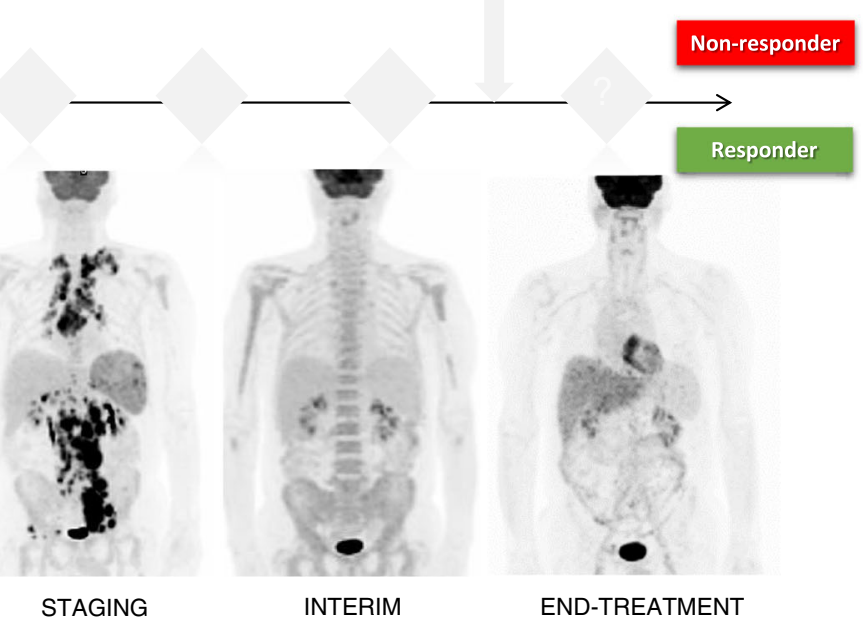

response at interim, with residual active disease in the abdomen (red circle; DS 5) and a subsequent progression at end-treatment evaluation. Patient \#2 (lower panels), despite the extensive disease at baseline, displays a complete metabolic response at interim (DS 1), confirmed at endtreatment evaluation
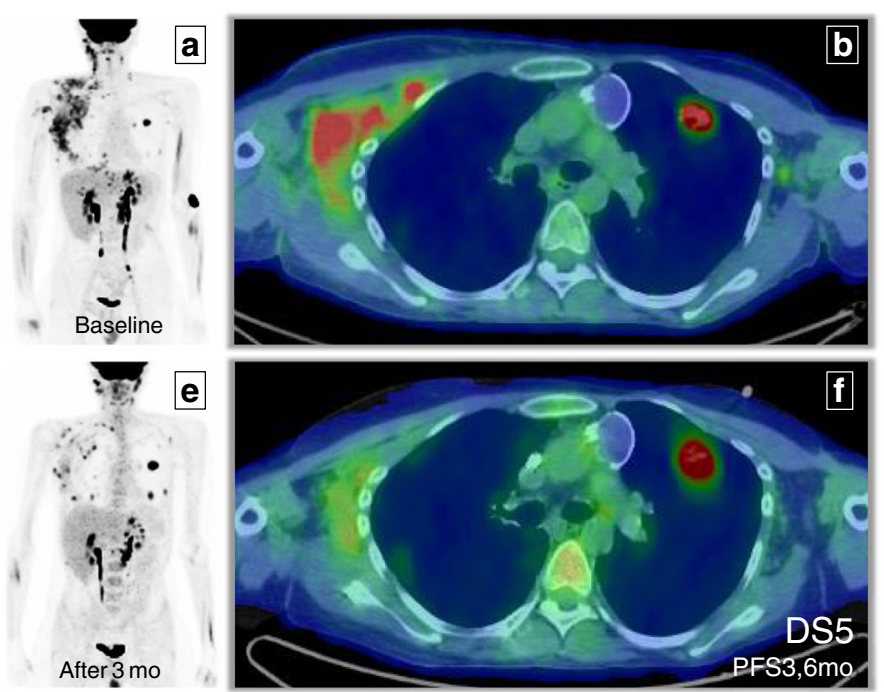

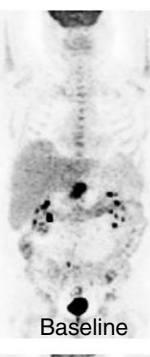

c
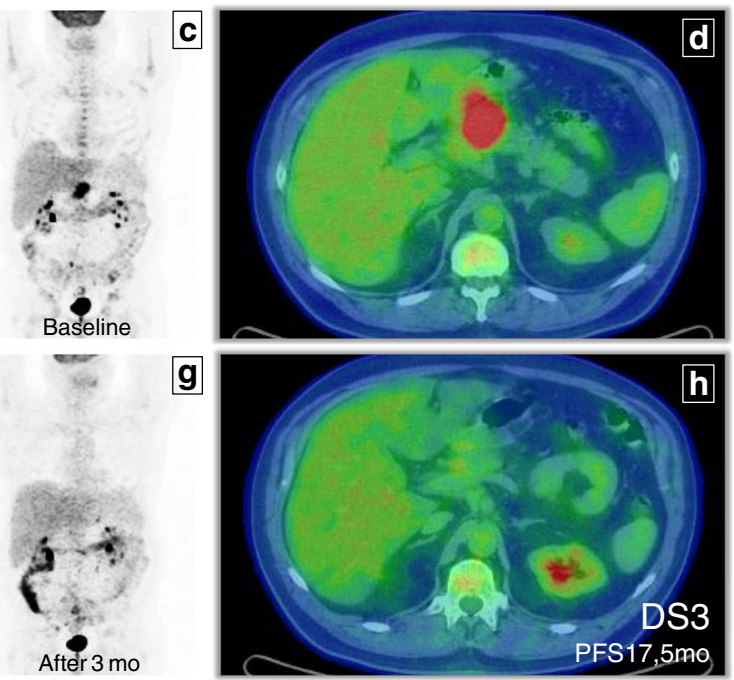

Fig. 2 Example of patients with Hodgkin's lymphoma undergoing immunotherapy with Nivolumab investigated at baseline (a-d) and after 3 months of treatment $(\mathbf{e}-\mathbf{h})$. These two cases display different patterns of response: patient \#1 (a, b, e, f) shows a mixed progressive disease with DS 5, which impacts negatively on the outcome (progression-free survival: PFS 3.6 months); patient \#2 (c, d, g, h) results a complete metabolic responder with DS 3 and a significantly longer PFS (17.5 months) than previously 
regimens, Deauville score, like the mythological Phoenix, rises from its ashes.

Can we state that the game is completed? Absolutely, not! Large-scale prospective trials, designed as well as done by Kobe and colleagues, are mandatory. Moreover, as expert opinion recommendations propose, the straightforward visual analysis with Deauville score must be assessed alongside the possibility of "pseudo-progression", the occurrence of immune-related side effects and the right moment for response assessment [7, 12].

Acknowledgements The Italian Association for Research on Cancer (AIRC - Associazione Italiana per la Ricerca sul Cancro) is acknowledged for the support of this research.

\section{Compliance with ethical standards}

All procedures performed in studies involving human participants were in accordance with the ethical standards of the institutional and/or national research committee and with the 1964 Helsinki Declaration and its later amendments or comparable ethical standards.

Conflicts of interest E.L. is supported with the grant no. 18,923 provided by AIRC.

\section{References}

1. Borchmann $\mathrm{P}$, Goergen $\mathrm{H}$, Kobe $\mathrm{C}$, et al. PET-guided treatment in patients with advanced-stage Hodgkin's lymphoma (HD18): final results of an open-label, international, randomised phase 3 trial by the German Hodgkin study group. Lancet. 2018;390(10114):2790802.

2. Casasnovas O, Brice P, Bouabdallah R, et al. Randomized phase III study comparing an early PET driven treatment de-escalation to a not PET-monitored strategy in patients with advanced stages Hodgkin lymphoma: Final analysis of the AHL2011 LYSA study. Abstract 7503. 2018 ASCO Annual Meeting, Chicago.

3. Meignan M, Gallamini A, Meignan M, et al. Report on the first international workshop on interim-PET scan in lymphoma. Leuk Lymphoma. 2009;50:1257-60.

4. Kobe C, Goergen H, Baues C, et al. Outcome-based interpretation of early interim PET in advanced-stage Hodgkin lymphoma. Blood. 2018. https://doi.org/10.1182/blood-2018-05-852129.

5. Gallamini A, Barrington SF, Biggi A, et al. The predictive role of interim positron emission tomography for Hodgkin lymphoma treatment outcome is confirmed using the interpretation criteria of the Deauville five-point scale. Haematologica. 2014;99(6):110713.

6. Meignan M, Barrington S, Itti E, et al. Report on the 4th international workshop on positron emission tomography in lymphoma held in Menton, France, 3-5 October 2012. Leuk Lymphoma. 2014;55(1):31-7.

7. Cheson BD, Ansell S, Schwartz L, et al. Refinement of the Lugano classification lymphoma response criteria in the era of immunomodulatory therapy. Blood. 2016;128:2489-96.

8. Younes A, Hilden P, Coiffier B, et al. International working group consensus response evaluation criteria in lymphoma (RECIL 2017). Ann Oncol. 2017;28(7):1436-47.

9. Dercle L, Seban RD, Lazarovici J, et al. 18F-FDG PET and CT scans detect new imaging patterns of response and progression in patients with Hodgkin lymphoma treated by anti-programmed death 1 immune checkpoint inhibitor. J Nucl Med. 2018;59:15-24.

10. Castello A, Grizzi F, Qehajaj D, Rahal D, Lutman F, Lopci E. 18FFDG PET/CT for response assessment in Hodgkin lymphoma undergoing immunotherapy with checkpoint inhibitors. Leuk Lymphoma. 2018;22:1-9.

11. Rossi C, Gilhodes J, Maerevoet M, et al. Efficacy of chemotherapy or chemo-anti-PD-1 combination after failed anti-PD-1 therapy for relapsed and refractory Hodgkin lymphoma: a series from Lysa centers. Am J Hematol. 2018. https://doi.org/10.1002/ajh.25154.

12. Nanni C, Cottereau AS, Lopci E, et al. Report of the 6th international workshop on PET in lymphoma. Leuk Lymphoma. 2017;58(10):2298-303. 